\title{
Latest results of the Pierre Auger Observatory and astrophysical interpretations
}

\author{
Kumiko Kotera $^{a *}$ and Patrick Younk ${ }^{b *}$, for the Pierre Auger Collaboration ${ }^{c, d}$ \\ ${ }^{a}$ Department of Astronomy \& Astrophysics, Enrico Fermi Institute, and Kavli Institute for \\ Cosmological Physics, The University of Chicago, Chicago, Illinois 60637, USA. \\ ${ }^{b}$ Department of Physics, The University of Siegen, 57072 Siegen, Germany \\ ${ }^{c}$ Av. San Martín Norte 304 (5613) Malargüe, Prov. de Mendoza, Argentina \\ ${ }^{d}$ A full author list and affiliations can be found at \\ http://www.auger.org/archive/authors_2011_01.html. \\ E-mail: kotera@uchicago.edu, pwyounk@gmail.com
}

The Pierre Auger Observatory was designed to detect ultrahigh energy cosmic rays (UHECRs) and to unveil some of the long-standing issues associated with these particles, such as their origin, their acceleration mechanisms, and their interactions. Recent measurements made with the Pierre Auger Observatory of the flux, arrival directions, and composition of UHECRs are presented. Upper limits on the high energy neutrino and photon flux are also presented. The physical and astrophysical interpretations of these measurements are discussed.

25th Texas Symposium on Relativistic Astrophysics

December 6-10, 2010

Heidelberg, Germany

\footnotetext{
* Speaker.
} 


\section{Introduction}

The origin of ultra-high energy cosmic rays (UHECRs) is still unknown, despite decades of theoretical and experimental endeavor (see [1,2] for reviews). Progress in this field is hindered by major astrophysical and physical issues. Cosmic rays are charged particles and are deflected during their propagation in the magnetized universe - how our universe is magnetized being another obscure point of astrophysics. Our poor knowledge on the most powerful astrophysical objects, which are likely the sources of UHECRs, is another obstacle to understand how these particles can be produced. Furthermore, UHECRs reach energies that cannot be reproduced in the laboratory. Thus, the signatures that a UHECR produces in a detector have significant systematic uncertainties.

The low flux of UHECR requires detectors with large exposure in order to reach acceptable statistics. Completed in 2008, the Pierre Auger Observatory is the largest aperture cosmic ray observatory at present [3]. Constructed in the province of Mendoza, Argentina, by a collaboration of 18 countries, it measures the extensive air showers initiated by primary cosmic rays. The observatory consists of a $3,000 \mathrm{~km}^{2}$ array of water Cherenkov stations with $1.5 \mathrm{~km}$ spacing on a triangular grid (the surface detector, SD), overlooked by 24 fluorescence telescopes housed in four buildings (fluorescence detector, FD). The two detectors are highly complementary. The FD has better energy resolution and well understood energy sytematics, while the SD has greater statistics (nearly $100 \%$ on-time compared to $\sim 10 \%$ for the FD) and a well defined aperture. Furthermore, the geometrical reconstruction of hybrid events (detected with the FD and at least one SD station) is much more accurate compared to an event detected with the FD alone.

We first present the latest measurements of the Pierre Auger Observatory with regard to the energy spectrum, the arrival directions, and the composition of UHECR. Then, we discuss the interpretation of each of these results by comparing them with the theoretical expectations. We conclude with a discussion of the ongoing and future search plans for UHECR sources.

\section{Measurements}

\subsection{Energy spectrum}

A key observable in the study of UHECRs is their energy spectrum measured on Earth. Figure 1 presents the energy spectrum calculated using data from the Pierre Auger Observatory [4]. This spectrum was obtained by combining the spectra derived from SD events and from hybrid events, using a maximum likelihood method. This analysis used SD events acquired between January 2004 and December 2008 and hybrid events acquired between November 2005 and May 2008.

The spectrum can be described by a broken power-law, $E^{-s}$, with spectral index $s \sim 3.3$ below the break (called the "ankle") around $10^{18.6} \mathrm{eV}$, and $s \sim 2.6$ above, followed by a flux suppression above $\sim 10^{19.5} \mathrm{eV}$. The vertical error bars represent statistical errors. The systematic error on the absolute energy scale is about $22 \%$. The spectrum from the HiRes experiment [5], also shown in Figure 1, is consistent with the Auger spectrum within this systematic error.

\subsection{Arrival directions}

The 13 events collected by the Pierre Auger Observatory between 27 May 2006 and $31 \mathrm{Au}-$ gust 2007 with energies above $55 \mathrm{EeV}$ are not consistent with the hypothesis of isotropic arrival 


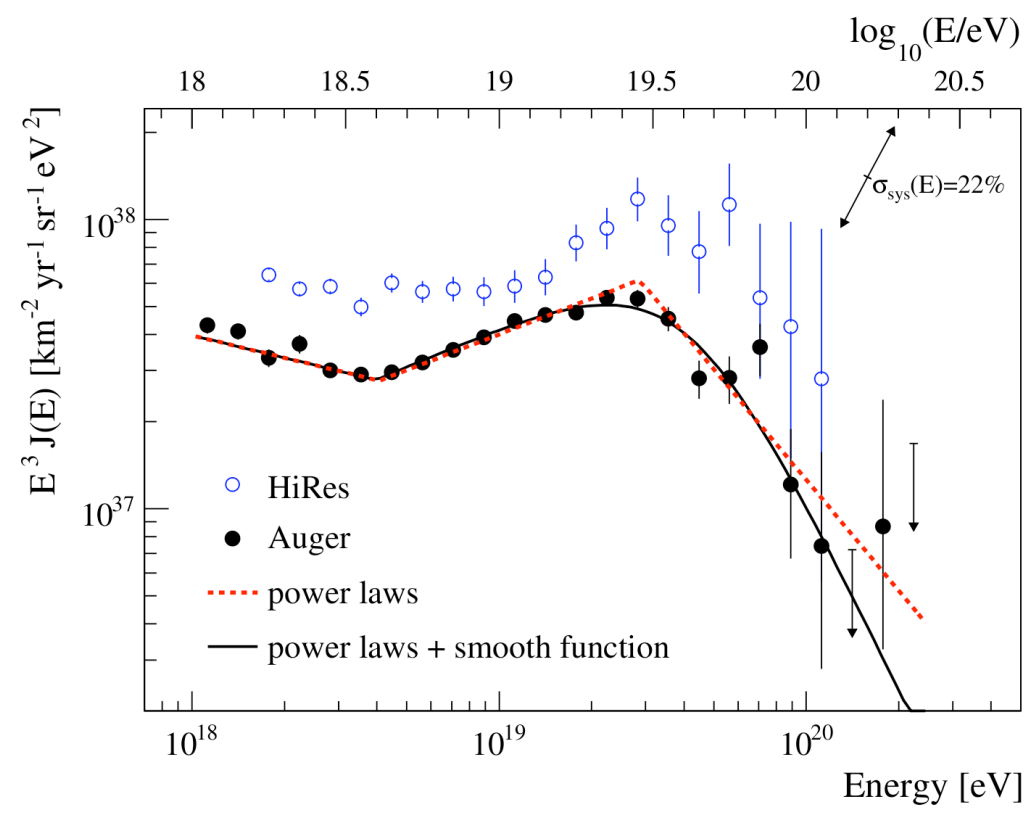

Figure 1: The energy spectrum measured by the Pierre Auger Observatory [4]. The spectrum measured by the HiRes experiment [5] is also shown for comparison.

directions [6, 7]. The hypothesis test measured the fraction of events with energy above $55 \mathrm{EeV}$ that were less than $3.1^{\circ}$ from the position of an AGN within $75 \mathrm{Mpc}$ in the VCV catalog [8]. The test parameters were set a priori, and they were optimized with an exploratory scan of the events collected prior to 27 May 2006.

Between 31 August 2007 (the completion of the hypothesis test) and 31 December 2009, 42 additional events were measured with energy greater than $55 \mathrm{EeV}$. For the data set that is the union of these 42 events and the 13 events used in the hypothesis test, the fraction of correlating events is $38_{-6}^{+7} \%$ [9]. If the cosmic ray arrival directions are isotropically distributed, a correlating fraction of $21 \%$ is expected. Figure 2 shows a sky map of the 69 events above $55 \mathrm{EeV}$ measured up to 31 December 2009, including the 14 events used in the exploratory scan.

\subsection{Chemical composition}

The atmospheric depth of shower maximum $X_{\max }$ is well correlated with the mass of the primary cosmic ray. On average the shower maximum for protons occurs deeper in the atmosphere than that for the same energy iron nucleus. In addition, the natural shower-to-shower fluctuations (i.e., the width of the $X_{\max }$ distribution, $\operatorname{RMS}\left(X_{\max }\right)$ ) for proton primaries are greater than for iron primaries. Figure 3 shows the average $X_{\max }$ and $\operatorname{RMS}\left(X_{\max }\right)$ vs. energy for high quality hybrid events measured with the Pierre Auger Observatory [10]. Monte Carlo simulations for protons and iron nuclei using different hadronic interaction models are also shown. The spread of the predictions for the the different models demonstrates that the systematic uncertainties in this analysis can be significant.

A total of 3754 events above $1 \mathrm{EeV}$ was used in the analysis. Fiducial cuts were applied to ensure an unbiased sampling of the actual $X_{\max }$ distribution. The $X_{\max }$ resolution above $3 \times 10^{18} \mathrm{eV}$ 


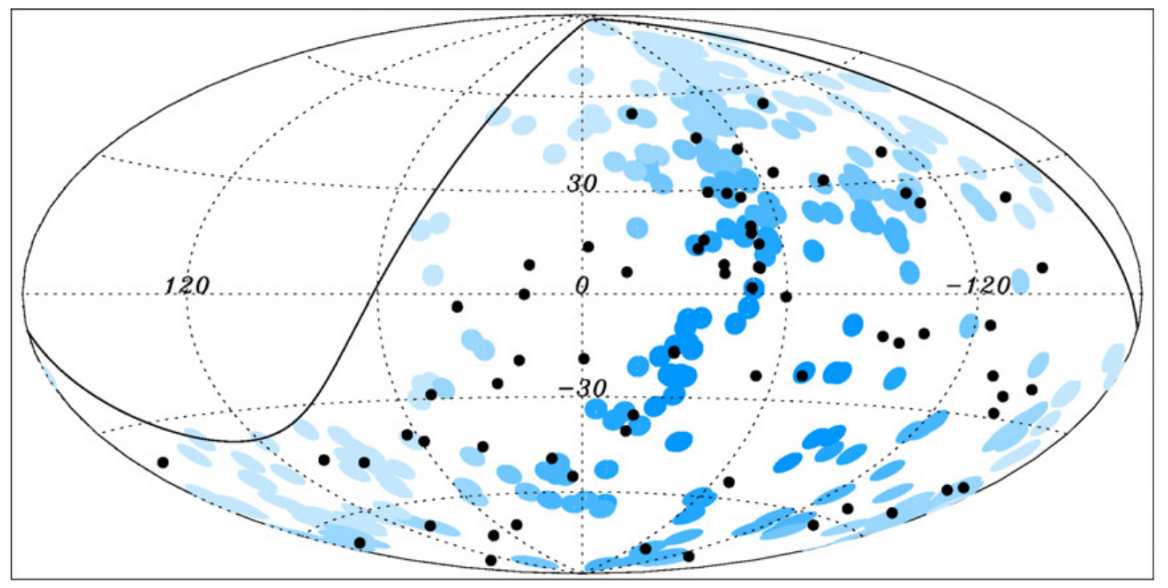

Figure 2: The 69 arrival directions of CRs with energy greater than $55 \mathrm{EeV}$ detected by the Pierre Auger Observatory up to 31 December 2009 are plotted as black dots in an Aitoff- Hammer projection of the sky in galactic coordinates. The solid line represents the border of the field of view for zenith angles smaller than $60^{\circ}$. Blue circles of radius $3.1^{\circ}$ are centered at the positions of the 318 AGNs in the VCV catalog that lie within $75 \mathrm{Mpc}$ and that are within the field of view of the Observatory. Darker blue indicates larger relative exposure. From Ref. [9].
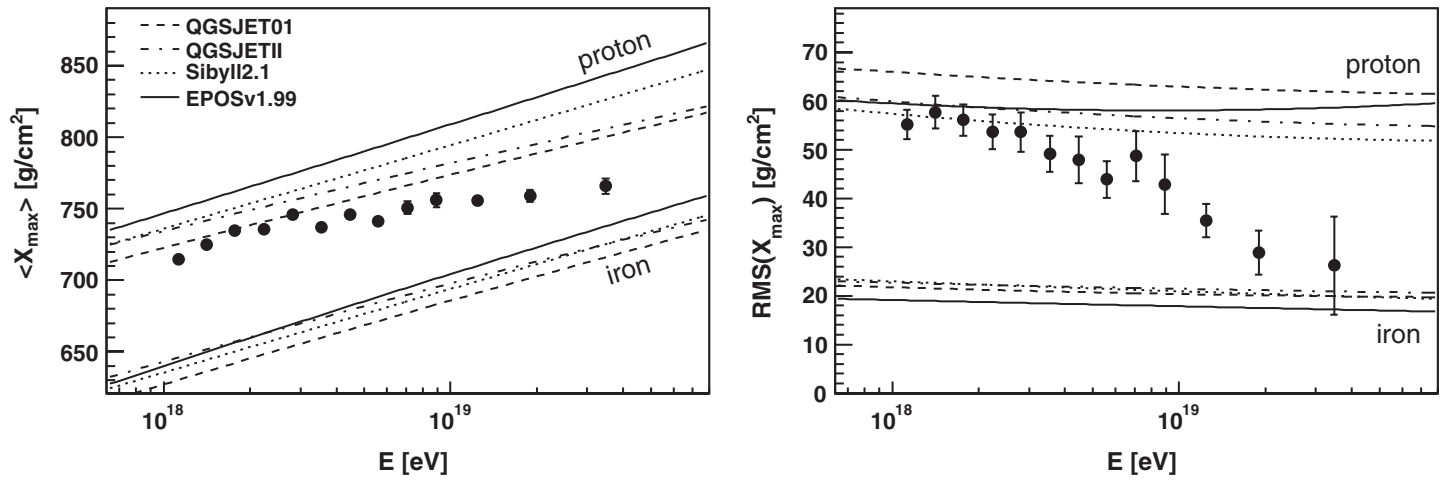

Figure 3: $\left\langle X_{\max }\right\rangle$ and $\operatorname{RMS}\left(X_{\max }\right)$ as a function of energy [10]. Monte Carlo simulations using different hadronic interaction models are displayed for protons and iron nuclei.

is $20 \mathrm{~g} / \mathrm{cm}^{2}$. At $\sim 1 \mathrm{EeV}$, the measurements seem consistent with light primaries. At higher energies (up to $40 \mathrm{EeV}$ ), both $\left\langle X_{\max }\right\rangle$ and $\operatorname{RMS}\left(X_{\max }\right)$ results favor a trend toward heavier primaries.

\subsection{Neutrinos and photons}

Photons and earth skimming tau neutrinos are expected to produce air showers with unique characteristics. Photons are expected to produce an air shower with a relatively deep $X_{\max }$ as measured by the FD. Both photons and earth skimming tau neutrinos are expected to produce showers that have signatures in the SD that are collectively described as a "young shower", with the showers initiated by earth skimming tau neutrinos being nearly horizontal. By searching for air showers with these characteristics, limits on the flux of ultra-high energy photons and tau neutrinos 
can be obtained.

The current upper limit set with Auger data for the fraction of photons in the UHECR flux is $2 \%$ above $10 \mathrm{EeV}$ and $2.4 \%$ above $2 \mathrm{EeV}[11,12]$. The current upper limit set with Auger data for the tau neutrino flux is $6 \times 10^{-8} \mathrm{GeV} \mathrm{cm} \mathrm{s}^{-1} \mathrm{sr}^{-1}$ in the energy range $0.2 \mathrm{EeV}$ to $20 \mathrm{EeV}$, with the assumption that the spectrum is proportional to $E^{-2}$ [13].

\section{Discussion}

\subsection{Energy spectrum: source and propagation signatures}

The shape of the energy spectrum observed by Auger supports the long-held notion that sources of UHECRs are extragalactic. The steep decline in flux above about $30 \mathrm{EeV}$ appears very much like that expected from the effect of interactions between extragalactic cosmic rays and the cosmic background radiation, named the Greisen-Zatsepin-Kuzmin (GZK) cutoff [14, 15]. A similar cutoff could however be produced by a maximum acceleration energy $E_{\max }$ at the source. Another important feature is the hardening of the spectrum at a few EeV (the ankle), which may be caused by the transition from Galactic to extragalactic cosmic rays or by propagation losses if UHECRs are mostly protons. The theoretically calculated spectra are in very good agreement with the observed spectra for a variety of chemical compositions, galactic to extragalactic transition models, source evolution histories, and injection spectrum indices. Better constraints on the systematic and statistical uncertainties will be needed to converge toward a clear scenario using the shape of the energy spectrum.

The requirements for astrophysical objects to accelerate UHECRs are quite stringent, and only a few candidates, such as very powerful active galactic nuclei, long gamma-ray bursts, and newly born magnetars, meet these requirements [1].

\subsection{Arrival directions and magnetic fields}

Inhomogeneities in the spatial distribution of the candidate sources within the GZK horizon may imprint a detectable anisotropy in the UHECR arrival directions. A correlation of arrival directions with nearby matter could be expected for high energy protons because it is plausible that the magnetic deflection of these particles is much less than 10 degrees. However, heavy nuclei are likely to deviate more than 10 degrees from their sources.

The arrival directions of cosmic rays above $55 \mathrm{EeV}$ appear to be correlated with nearby extragalactic matter. The isotropy test [6] was conducted with a template derived from the positions of AGN within $75 \mathrm{Mpc}$ listed in the VCV catalog [8]. Furthermore, a posteriori statistical tests [9] have shown a correlation with nearby galaxies in the 2MRS catalog and with nearby AGN detected by Swift-BAT. Better statistics will provide a clearer view of the role of the cosmic magnetic fields and could allow a discrimination of possible sources.

\subsection{Challenging composition measurements?}

Between $10^{18}$ and $10^{19} \mathrm{eV}$, measurements of the depth of shower maximum are consistent with a significant fraction of protons in the cosmic ray flux. Above $10^{19} \mathrm{eV}$, however, measurements of shower development are challenging to explain if a significant proton fraction is present. In 
particular, the shower-to-shower fluctuations observed above $10^{19} \mathrm{eV}$ favor a heavier composition with a relatively small proton component. A knowledge of the cosmic-ray composition is important for deciding which of several source scenarios is more likely.

Changes to hadronic interactions from current extrapolations provide a plausible alternative interpretation to the observed shower development behavior. The relevant center-of-mass energy for a UHECR proton interacting in the atmosphere can be near $100 \mathrm{TeV}$, while highest energy hadronic interactions observed in the laboratory are $7 \mathrm{TeV}$. The observation of anisotropies and secondary particles (neutrinos and gamma-rays) can lead to astrophysical constraints on the composition of UHECRs, opening the possibility for the study of hadronic interaction cross sections, multiplicities, and other interaction parameters at hundreds of $\mathrm{TeV}$.

\subsection{Secondary messengers: neutrinos and photons}

Secondary neutrinos and photons can be produced by UHECRs when they interact with ambient baryonic matter and radiation fields inside the source or during their propagation from source to Earth. These particles travel in geodesics mostly unaffected by magnetic fields and bear valuable information of the birthplace of their progenitors.

Limits on the photon fraction place stringent limits on models where UHECRs are generated by the decay of super heavy dark matter and topological defects. Unfortunately, the uncertainties on the UHECR source composition, spectrum, and redshift evolution translates to many orders of magnitude uncertainty in the expected cosmogenic neutrino flux as discussed in [16] and references therein. It is interesting to notice however that the strongest source evolution models with pure proton composition are already being ruled out by the Auger limits.

\section{Perspectives: What will be needed}

The precise measurements and large statistics of the Pierre Auger Observatory have significantly increased our knowledge of UHECRs. Looking forward, decreasing the statistical and systematic uncertainty of the three pillars of UHECR observations (spectrum, arrival direction distribution, and composition) is the key to solving at last the mystery of the origins of UHECRs.

The precise shape and energy scale of the ankle and the cutoff are excellent selectors of models. More discriminating, but much harder to plan for success, is the appearance of clear structure in the arrival directions. A nearby source, the first UHECR source, will be a watershed in the field [17]. Also very discriminating is a clear indication of the composition. The dependence on hadronic models to translate shower properties into composition measurements makes it difficult to reach clear conclusions (i.e., the limiting factor is systematic uncertainties). Progress on increasing the number of high quality measurements in the energy range between the knee and the ankle (e.g., the HEAT and AMIGA enhancements to the Pierre Auger Observatory) will help construct a unified model of the cosmic ray properties in a region that hadronic models can be tested at the LHC ${ }^{1}$. Finally, observations of photons and neutrinos at ultrahigh energies will also be extremely useful in distinguishing proposed scenarios.

\footnotetext{
${ }^{1}$ see http://www-ik.fzk.de/ needs/ for a list of measurements that can be made at accelerator programs to improve the ability of hadronic models to interpret cosmic ray observables.
} 


\section{Acknowledgments}

PY would like to thank the Alexander von Humboldt Foundation for financial support. KK acknowledges support by the NSF grant PHY-0758017 at the University of Chicago, and the Kavli Institute for Cosmological Physics through grant NSF PHY-0551142 and an endowment from the Kavli Foundation.

\section{References}

[1] K. Kotera and A. V. Olinto. The Astrophysics of Ultrahigh Energy Cosmic Rays. ARAA, 49, 2011.

[2] A. Letessier-Selvon and T. Stanev. Ultrahigh energy cosmic rays. Rev. Mod. Phys., 2011.

[3] J. Abraham et al. Properties and performance of the prototype instrument for the Pierre Auger Observatory. Nucl. Instrum. Meth. in Phys. Res. A, 523:50-95, May 2004.

[4] J. Abraham et al. Measurement of the energy spectrum of cosmic rays above $10^{18} \mathrm{eV}$ using the Pierre Auger Observatory. Physics Letters B, 685:239-246, March 2010.

[5] R. U. Abbasi et al. Measurement of the flux of ultra high energy cosmic rays by the stereo technique. Astroparticle Physics, 32:53-60, August 2009.

[6] J. Abraham et al. Correlation of the Highest-Energy Cosmic Rays with Nearby Extragalactic Objects. Science, 318:938, November 2007.

[7] J. Abraham et al. Correlation of the highest-energy cosmic rays with the positions of nearby active galactic nuclei. Astroparticle Physics, 29:188-204, April 2008.

[8] M.-P. Véron-Cetty and P. Véron. A catalogue of quasars and active nuclei: 12 th edition. $A \& A$, 455:773-777, August 2006.

[9] P. Abreu et al. Update on the correlation of the highest energy cosmic rays with nearby extragalactic matter. Astroparticle Physics, 34:314, September 2010.

[10] J. Abraham et al. Measurement of the Depth of Maximum of Extensive Air Showers above $10^{1} 8 \mathrm{eV}$. Phys. Rev. Lett., 104:091101, 2010.

[11] J. Abraham et al. Upper limit on the cosmic-ray photon fraction at eev energies from the pierre auger observatory. Astroparticle Physics, 31:399, 2009.

[12] J. Abraham et al. Upper limit on the cosmic-ray photon flux above $10^{19} \mathrm{ev}$ using the surface detector of the pierre auger observatory. Astroparticle Physics, 29:243, 2008.

[13] J. Abraham et al. Limit on the diffuse flux of ultrahigh energy tau neutrinos with the surface detector of the pierre auger observatory. Physical Review D, 79:102001, 2009.

[14] K. Greisen. End to the cosmic-ray spectrum? Phys. Rev. Lett., 16:748-750, 1966.

[15] G.T. Zatsepin and V.A. Kuzmin. Upper limit of the spectrum of cosmic rays. J. Exp. Theor. Phys. Lett., 4:78-80, 1966.

[16] K. Kotera, D. Allard, and A. V. Olinto. Cosmogenic neutrinos: parameter space and detectabilty from PeV to ZeV. JCAP, 10:13, October 2010.

[17] P. W. Younk. Estimating the flux of the brightest cosmic-ray source above $57 \times 10^{18}$ ev. Astrophysical Journal, 696:L40, 2009. 